\title{
GEOSTATIONARY ATMOSPHERIC SOUNDING' BY FORMATION FLIGHT APERTURE SYNTHESIS
}

\author{
Ahmed Kiyoshi Sugihara El Maghraby*,1, Angelo Grubišić ${ }^{*}$, Camilla Colombo ${ }^{2}$, Adrian Tatnall ${ }^{1}$ \\ ${ }^{1}$ University of Southampton, UK, ${ }^{2}$ Politecnico di Milano, Italy \\ *asem1g14@soton.ac.uk
}

\begin{abstract}
-
This paper introduces a multi-satellite approach to passive microwave interferometric radiometry applied to geostationary atmospheric sounding at $53 \mathrm{GHz}$. The concept applies satellite formation flight to the currently operational interferometric techniques to extend the achievable microwave aperture sizes, leading to unprecedented spatial resolution for microwave radiometers. The presented configurations are capable of synthesising $14.4 \mathrm{~m}$ aperture and larger using SMOS-sized satellites, and spatial resolution better than $16.7 \mathrm{~km}$ at $53 \mathrm{GHz}$ from the geostationary orbit. Two instrument concepts are proposed: Single-Element Companion concept and Array Duplicate concept, both of which are scalable, where the achievable spatial resolution is extendible and constrained only by the number of satellites in the constellation. The performance of both concepts are simulated, and the results show that the interferometer is highly sensitive to uncertainties in inter-satellite position measurements, where measurement errors as small as 6 $\mathrm{mm}$ result in $6.3 \mathrm{~K}$ root mean square error in radiometric accuracy.
\end{abstract}

\section{INTRODUCTION}

While passive microwave sounders are highly versatile tool for meteorology and Earth observation, providing atmospheric temperature, humidity and precipitation measurements, their poor spatial resolution have bound these instruments to the low Earth orbit. In recent years the geostationary orbit has been in consideration as a desirable orbit for future atmospheric sounders, in order to achieve global and real-time observation. [1] Subsequently several interferometric techniques are currently being developed by NASA, ESA and China, with radiometer concepts GeoSTAR [2], GAS [3] and GIMS [4] respectively, that would for the first time enable radiometry from the geostationary orbit at $53 \mathrm{GHz}$ for temperature sounding, and higher for humidity. Since these concepts are designed around a single satellite platform however, their achievable aperture size is fundamentally constrained by the satellite's physical size. To access any lower sounding frequencies, or to further improve the spatial resolution, we propose a multi-satellite approach to overcome this physical constraint. The proposed concept uses a set of interferometer antenna elements mounted on freeflying satellites. The signals from these elements are wirelessly transmitted to the relevant satellites where signal cross-correlation is performed. The concept's spatial resolution is determined by the longest intersatellite separation distance, while the shape of the synthesised aperture is determined by the geometry of the constellation. The proposed formation geometries are capable of synthesising scalable, circular apertures for circular beams. These configurations and their performance are now described. 


\begin{tabular}{llll}
\hline Comps & $\begin{array}{l}\text { Aperture } \\
\text { Diameter }\end{array}$ & $\begin{array}{l}\text { Spatial } \\
\text { Resolution }\end{array}$ & Mass \\
\hline 3 & $2.0 R=8.0 \mathrm{~m}$ & $30.1 \mathrm{~km}$ & $868 \mathrm{~kg}$ \\
9 & $3.6 R=14.4 \mathrm{~m}$ & $16.7 \mathrm{~km}$ & $1288 \mathrm{~kg}$ \\
18 & $5.0 R=20.0 \mathrm{~m}$ & $12.0 \mathrm{~km}$ & $1918 \mathrm{~kg}$ \\
30 & $6.5 R=26.2 \mathrm{~m}$ & $9.2 \mathrm{~km}$ & $2758 \mathrm{~kg}$ \\
45 & $8.0 R=32.0 \mathrm{~m}$ & $7.5 \mathrm{~km}$ & $3808 \mathrm{~kg}$ \\
\hline
\end{tabular}

(a) Single-Element Companion

\begin{tabular}{llll}
\hline Sats & $\begin{array}{l}\text { Aperture } \\
\text { Diameter }\end{array}$ & $\begin{array}{l}\text { Spatial } \\
\text { Resolution }\end{array}$ & Mass \\
\hline 3 & $4.0 R=16.0 \mathrm{~m}$ & $15.0 \mathrm{~km}$ & $1974 \mathrm{~kg}$ \\
6 & $7.2 R=28.8 \mathrm{~m}$ & $8.4 \mathrm{~km}$ & $3948 \mathrm{~kg}$ \\
9 & $10.0 R=40.0 \mathrm{~m}$ & $6.0 \mathrm{~km}$ & $5922 \mathrm{~kg}$ \\
12 & $13.1 R=52.5 \mathrm{~m}$ & $4.6 \mathrm{~km}$ & $7896 \mathrm{~kg}$ \\
15 & $16.0 R=64.0 \mathrm{~m}$ & $3.8 \mathrm{~km}$ & $9870 \mathrm{~kg}$ \\
\hline
\end{tabular}

(b) Array Duplicate

TABLE I: The scaling and system mass of the Single-Element Companion concept (a) and the Array Duplicate concept (b). The effective aperture with boom length $R=4 \mathrm{~m}$ on the central array. Total constellation mass is estimated with SMOS-sized central satellite at $658 \mathrm{~kg}$ and $70 \mathrm{~kg}$ companion microsatellites.

\section{Multi-SATELlite INTERFEROMETRY}

\section{CONCEPTS}

Two suitable formation flight configurations are identified and contrasted for the radiometer concept, both synthesising circular apertures. The first type is the Single-Element Companion, where a single fullsized satellite is flown in formation with several "companion" satellites, while the second type is the Array Duplicate, where several full-sized satellites are flown in formation.

Figure 1a shows the nine-companion configuration of the Single-Element Companion concept, whereby a single rotating Y-shaped central array identical in dimensions to the MIRAS instrument on board the SMOS mission [5] is accompanied by nine single antenna elements each on board identical formationflying companion satellites, labelled A to I. Because all correlation can be performed in the central satellite, the companion satellites can be as small as a microsatellite. The size of the existing MIRAS instrument has been chosen as a baseline in order to adhere to currently feasible satellite structures. This configuration produces a circular effective aperture 3.6 times the length of the booms on the Y-shaped central array, synthesising $14.4 \mathrm{~m}$ effective aperture at MIRAS $4 \mathrm{~m}$ design. The effective aperture of this concept is scalable, as summarised in Table Ia where effective aperture, spatial resolution and estimated total constellation mass are listed for a given number of companion satellites.

Figure $1 \mathrm{~b}$ shows the six-satellite configuration of the Array Duplicate concept, where six identical rotating arrays the size of SMOS, labelled a to f, fly in formation to produce a larger aperture of 7.2 times the boom length. As with the Single-Element Companion concept, this concept is also scalable by increasing the number of satellites, as summarised in Table Ib.

The expected spatial resolution and radiometric accuracy has been simulated at $53 \mathrm{GHz}$ centre frequency from the geostationary orbit. With configuration shown in Figure 1a spatial resolution of $16.7 \mathrm{~km}$ is achieved, while Figure $1 \mathrm{~b}$ shows $8.3 \mathrm{~km}$. With an input image of modified GOES13 imagery data (Figure 1c), shown in Figure $1 \mathrm{~d}$ is the recovered image by the configuration shown in Figure 1a, showing radiometric accuracy of $0.1 \mathrm{~K}$ ignoring fringe-washing and hardware imperfections.

The results show that the proposed interferometer is highly sensitive to the uncertainties in the measurement of satellites' relative position. The results are summarised in Table II with measurement errors of $0.1 \lambda$ and $1 \lambda$ for both concepts. These are found from simulation results as shown in Figure 1e, the retrieved brightness temperature error of the SingleElement Companion concept with $1 \lambda$ measurement error, approximately $6 \mathrm{~mm}$ at $53 \mathrm{GHz}$. It is found however that the cause of these errors is the uncertainty in measurement, and not the physical deviation of the 
relative positions. Figure 1f shows better radiometric accuracy levels at $3.5 \lambda$ deviation, approximately $2 \mathrm{~cm}$ at $53 \mathrm{GHz}$, when the deviation is measured without uncertainties.

\section{CONCLUSION}

Two viable formation configurations have been found and explored for geostationary atmospheric sounding. The first configuration is the Single-Element Companion concept with a SMOS-sized rotating Yshaped interferometer flying in formation with several formation-flying microsatellites, with total constellation mass of under two tonnes, achieving spatial resolution of $16.7 \mathrm{~km}$ and better at $53 \mathrm{GHz}$, with apertures of $14.4 \mathrm{~m}$ and larger. The second concept is the Array Duplicate concept with several SMOS-sized satellites flying in formation. Spatial resolution of $8.3 \mathrm{~km}$ and better can be achieved, with apertures of $28.8 \mathrm{~m}$ and larger, while total constellation mass may exceed five tonnes.

The arrays have been found highly sensitive to the uncertainties in the inter-satellite relative position measurement, meaning inter-satellite ranging with micron-level precision and accuracy is instrumental for the geostationary mission. It is also found that intersatellite position may deviate by a few centimetres while maintaining array performance, as long as this deviation is measured to the required precision and accuracy.

\section{ACKNOWLEDGMENTS}

This research has been funded by the UK Engineering and Physical Sciences Research Council (EPSRC). Award Reference number 1503202

\section{REFERENCES}

[1] U. Klein, Chung-Chi Lin, N. Atkinson, J. Charlton and C. Philpot, "Future microwave radiometers in geostationary and medium earth orbit," IGARSS 2003. 2003 IEEE International Geoscience and Remote Sensing Symposium. Proceedings (IEEE Cat. No.03CH37477), 2003, pp. 2158-2160.

\begin{tabular}{lrlll}
\hline \multicolumn{3}{c}{ Deviation } & \multicolumn{2}{l}{ RMS Error } \\
\hline Nominal & 0 & $\lambda$ & $0.79 \mathrm{~K}$ & $0.12 \mathrm{~K}$ \\
Concept 1 & 0.1 & $\lambda$ & $1.78 \mathrm{~K}$ & $1.45 \mathrm{~K}$ \\
& 1 & $\lambda$ & $7.01 \mathrm{~K}$ & $6.32 \mathrm{~K}$ \\
Concept 2 & 0.1 & $\lambda$ & $0.90 \mathrm{~K}$ & $0.42 \mathrm{~K}$ \\
& $1 \lambda$ & $5.90 \mathrm{~K}$ & $5.32 \mathrm{~K}$ \\
\hline Concept 1 & 3.5 & $\lambda$ & $2.87 \mathrm{~K}$ & $0.95 \mathrm{~K}$ \\
Concept 2 & 3.5 & $\lambda$ & $3.35 \mathrm{~K}$ & $1.18 \mathrm{~K}$ \\
\hline
\end{tabular}

TABLE II: Results on simulation trials on retrieved brightness temperature error induced by inter-satellite position uncertainty as a function of uncertainty magnitude. The two error values given are within the full field of view, and within Earth disc respectively. Strong error present at horizon due to the Gibbs phenomenon. The last two rows show the errors at $3.5 \lambda$ deviation which are measured without uncertainties.

[2] Tanner, A. B.; Wilson, W. J.; Lambrigsten, B. H.; Dinardo, S.; Brown, S.; Kangaslahti, P.; Gaier, T.; Ruf, C.; Gross, S.; Lim, B.; Musko, S.; Rogacki S.; Piepmeier, J., "Initial Results of the Geostationary Synthetic Thinned Array Radiometer (GeoSTAR) Demonstrator Instrument," IEEE TRANSACTIONS ON GEOSCIENCE AND REMOTE SENSING, 2007.

[3] Christensen, J.; Carlström, A.; Ekstrom, H.; Emrich, A.; Embretsén, J.; de Maagt, P.; Colliander, A., "GAS: the Geostationary Atmospheric Sounder," in Geoscience and Remote Sensing Symposium, 2007. IGARSS 2007. IEEE International, vol., no., pp.223-226, 23-28 July 2007

[4] Hao Liu; Ji Wu; Shengwei Zhang; Jingye Yan; Lijie Niu; Cheng Zhang; Weiying Sun; Huiling Li; Bin Li, "The Geostationary Interferometric Microwave Sounder (GIMS): Instrument overview and recent progress," in Geoscience and Remote Sensing Symposium (IGARSS), 2011 IEEE International, vol., no., pp.3629-3632, 24-29 July 2011

[5] J. Font et al., "SMOS: The Challenging Sea Surface Salinity Measurement From Space," in Proceedings of the IEEE, vol. 98, no. 5, pp. 649-665, May 2010. 


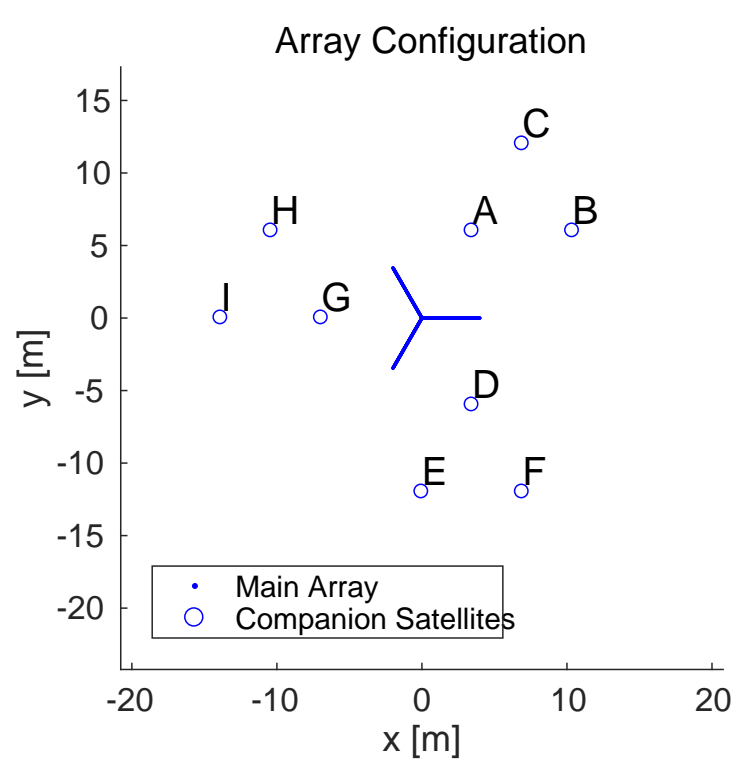

(a)

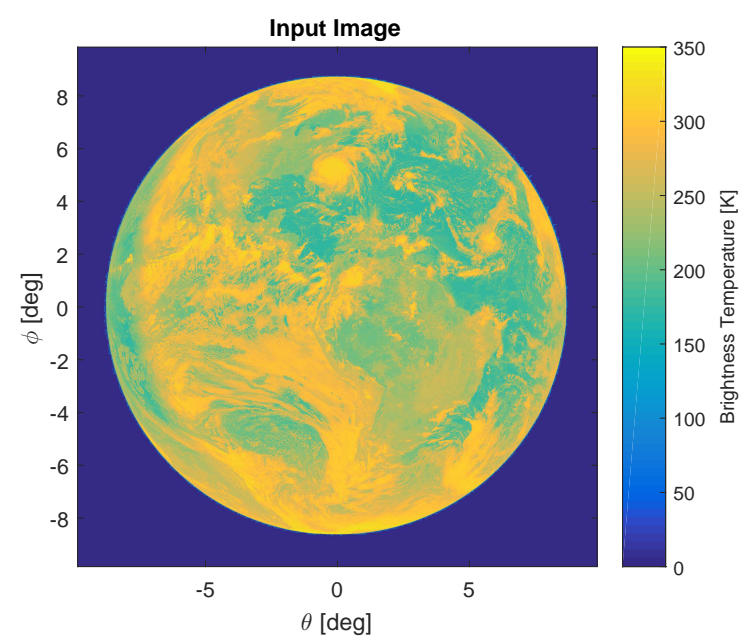

(c)

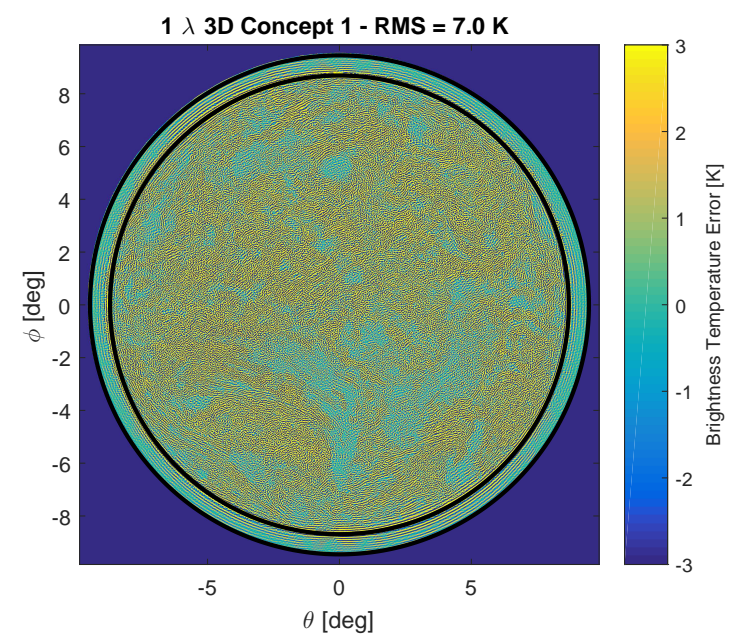

(e)

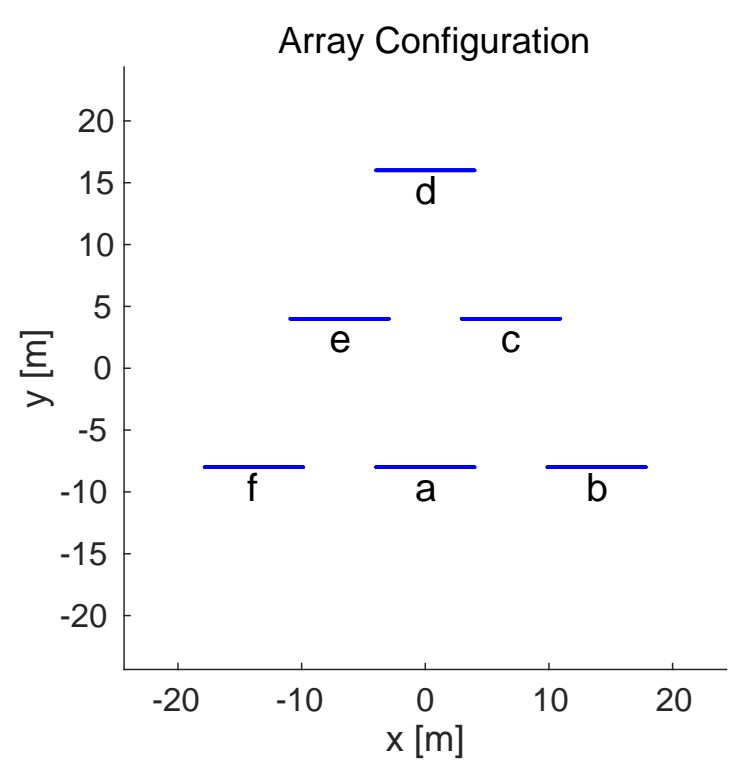

(b)

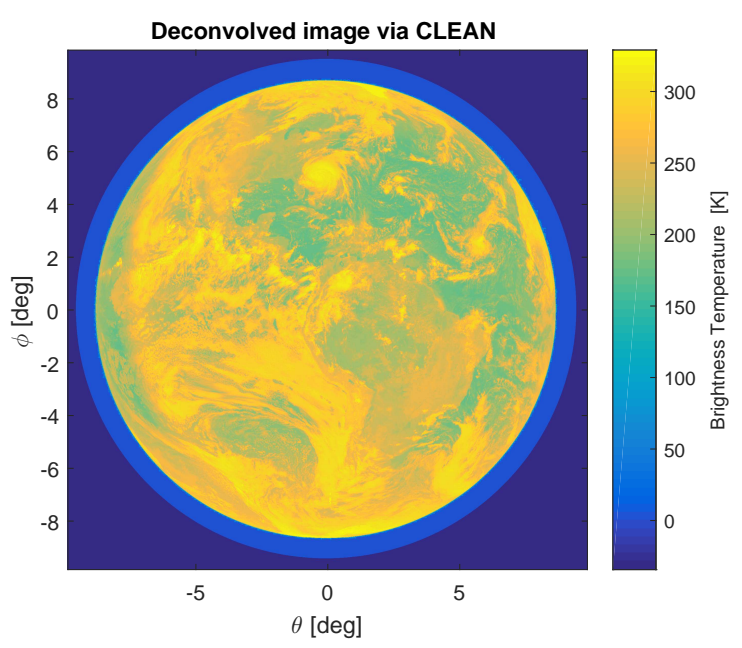

(d)

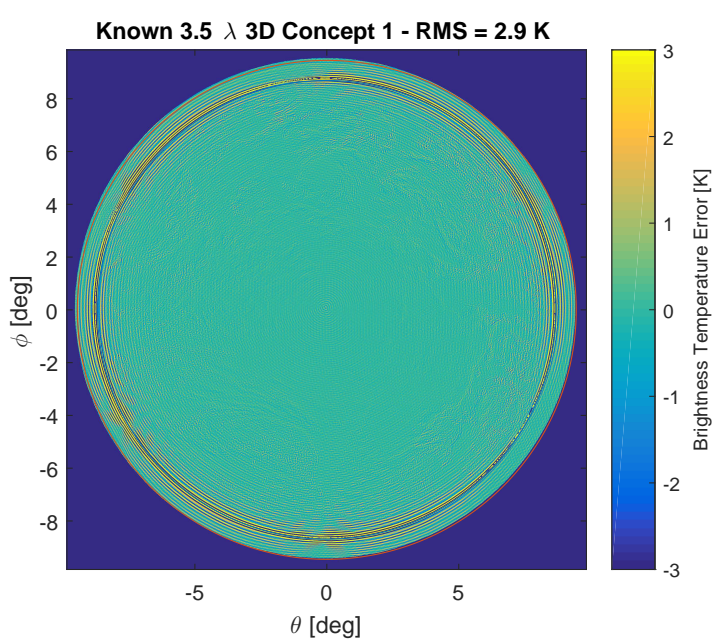

(f)

Fig. 1: Constellation layout for the Single-Element Companion concept where SMOS-sized central array flies in formation with nine microsatellites, labelled A to I (a). Array Duplicate concept where six SMOS-sized array fly in formation, labelled a to f (b). Input image of the Earth from GEO (c), the image reconstructed by the Single-Element Companion interferometer at $53 \mathrm{GHz}$ (d). (e) shows the retrieved brightness temperature error when the inter-satellite position measurement is uncertain at one wavelength $(6 \mathrm{~mm}$ at $53 \mathrm{GHz})$, and (f) shows the error when the deviation is large at 3.5 wavelengths, but is measured without uncertainty. 